\title{
Effect of Fungicide Spray Cover on Botrytis Cinerea Infection in Grape Bunches
}

\author{
J.C. Brink, G. Holz and P.H. Fourie* \\ Department of Plant Pathology, Stellenbosch University, Private Bag X1, 7602 Matieland (Stellenbosch), South Africa \\ jcbrink@sun.ac.za, gh@sun.ac.za,phfourie@sun.ac.za
}

Submitted for publication: March 2006

Accepted for publication: May 2006

Key words: fluorescent pigment, digital image analysis

\begin{abstract}
The effect of fungicide spray cover on Botrytis cinerea infection in grape bunches was determined. Bunches were sprayed at pea size and bunch closure with different volumes of a mixture of fenhexamid and a yellow fluorescent pigment, and the percentage fluorescent pigment coverage on pedicels was determined. Bunches were subsequently dusted with dry airborne conidia of $B$. cinerea in a settling tower and incubated for $\mathbf{2 4} \mathrm{h}$ at high relative humidity $(\mathbf{9 8 \%})$. Infection was determined by estimating the amount of $B$. cinerea infections on susceptible bunch parts (pedicel, receptacle and rachis) with isolations onto paraquat (herbicide) and Kerssies (B. cinerea selective) mediums. Linear regressions for the part $\times$ stage combinations of percentage $B$. cinerea incidence on different bunch parts were fitted on mean coverage levels. An increase in spray cover caused linear reductions in levels of B. cinerea on bunch parts. Higher $\boldsymbol{B}$. cinerea incidences were recorded at pea size. Furthermore, higher $\boldsymbol{B}$. cinerea incidences were found on paraquat medium for both stages, than on Kerssies medium. The information gathered from this study will be used to facilitate future determination of minimum effective coverage levels for effective $B$. cinerea control in grape bunches.
\end{abstract}

Botrytis bunch rot management strategies are based on knowledge of epidemiology, infection pathways and inoculum ecology of the causal pathogen Botrytis cinerea in vineyards (Holz et al., 2003). In South Africa, weather conditions are usually favourable for colonisation of inflorescences and young bunches from bloom to late pea-size stage. Conidium numbers in air and at various positions in bunches are high until bunch closure, and then rapidly decrease to very low levels (Van Schoor, 2004). Furthermore, bunch parts are often dusted with pollen, which sustains the pathogen's growth on their surfaces. B. cinerea grows on senescent tissues, penetrates the stamens and invades their bases situated on the receptacle and the localised necrotic areas around the abscission layer of the shredded calyptra on the receptacle (Holz et al., 2003). It also readily colonises the rachises, laterals, pedicels, ovaries and developing berries.

Host resistance varies in the different bunch parts. Structural bunch parts are susceptible to infection during the younger stages, but resistance increases moderately as the season progresses. Conidia and germlings can survive for extended periods on structural bunch parts but do not survive for long on the surface of immature berry skins when latent infection occurs in the skin (Holz et al., 2003). B. cinerea symptom expression is furthermore predominantly associated with pedicels and the bases of berries under South African conditions (Coertze et al., 2001; Coertze \& Holz, 2002; Holz et al., 2003). This is also true for other fungi (Penicillium, Aspergillus, Alternaria, Mucor and Rhizopus spp.) that are commonly associated with bunch rot (Hewitt, 1974; Holz et al., 2003). The next prominent positions occupied by $B$. cinerea are rachises and laterals, and not berry cheeks. Alternaria alternata also penetrates rachises and pedicels through stomata and lenticels, and causes rot of cold-stored table grapes when subjected to stress conditions (Swart \& Holz, 1994; Thomas et al., 1988). Bunch density, turgor, berry rupture, wind and insect damage (Holz \& Volkmann, 2002) are factors that lead to stress at the pedicel and the berry-base. These factors can also lead to the seeping of berry juice, which plays a prominent role in disease development (Nair et al., 1988). In these cases, symptoms develop in the absence of humid conditions or free water (Holz et al., 2003). Berry surfaces are thus, contrary to the structural bunch parts, not covered by conidia or germlings between bunch closure and harvest (Van Schoor, 2004). These findings imply that the berry-pedicel attachment zone is underestimated in the development of bunch rot epiphytotics in grapevines (Holz et al., 2003).

The control of $B$. cinerea infection by chemical, cultural and biological means can only be achieved by reducing inoculum on susceptible bunch parts at the appropriate growth stage (Van Rooi $\&$ Holz, 2003). Chemical control remains the most effective strategy to reduce the incidence of grey mould in grapevines. Three preventative fungicide applications are recommended: firstly, between budding and pre-bloom, to protect susceptible inflorescences; secondly, during bloom to pea-size stage, to reduce inoculum in clusters and to prevent colonisation of floral debris;

*Corresponding author: E-mail: phfourie@sun.ac.za

Acknowledgements: The authors acknowledge the Deciduous Fruit Producers'Trust, Winetech, BASF, Bayer, Dow Agrosciences, DuPont, Syngenta, Nexus, Terason, UAP and Wenkem for financial assistance; Frikkie Calitz (ARC Biometry Unit, Stellenbosch) for statistical analyses; and JD Kirsten (Paarl) and Lukas de Wet (Worcester) for research material (grape bunches). 
and thirdly, at bunch closure, to reduce inoculum of $B$. cinerea at various positions of the inner bunch, especially for cultivars with tight bunches (Van Rooi \& Holz, 2003; Van Schoor, 2004).

Laboratory studies (Van Rooi, 2001) have shown that when fungicides are properly applied to the susceptible target sites in bunches, the amount of $B$. cinerea at the various sites within bunches is reduced, and infection and symptom expression are prevented at all growth stages. The same efficacy is, however, not achieved with the same fungicides when using conventional spraying methods in vineyards (Holz et al., 2003). This can largely be attributed to incorrect deposition of fungicides on the susceptible target sites. Higher volumes will generally lead to better coverage (Brink et al., 2004), but may lead to a higher risk of exceeding permitted maximum residue levels (Leroux, 1995) or to reduced coverage because of run-off. The optimisation of fungicide application in order to facilitate sufficient spray cover for effective disease management is therefore of utmost importance.

A variety of methods have been used to assess spray cover in vineyards, but none of these methods can quantify spray deposits on a very small, three-dimensional area of interest, such as the susceptible grape bunch parts (Holownicki et al., 2002). Furthermore, methods that are dependent on human discretion lack quantitative measuring and speed of measurement (Derkson \& Jiang, 1995). A spray coverage assessment protocol using fluorometry, photomicrography and digital image analyses was developed to accurately measure spray cover on susceptible grape bunch parts (Brink et al., 2004). The aim of this study was to use the protocol to determine the effect of different levels of spray cover in grape bunches on $B$. cinerea infection on different bunch parts.

\section{MATERIALS AND METHODS}

Grape bunches from the table grape cultivar Dauphine were selected at pea size and bunch closure stages from two vineyards in the Paarl and Worcester regions. Bunches were surface-sterilised before use (30 $\mathrm{s}$ in $70 \%$ ethanol, $2 \mathrm{~min}$ in $0.35 \%$ sodium hypochlorite, and $30 \mathrm{~s}$ in $70 \%$ ethanol) and air-dried.

\section{Experimental design}

The experimental design was a randomised block design with six spray volumes, replicated in five blocks. Each block was divided in four groups (treatment combinations) of six bunches each. Each bunch was sprayed with a different spray volume (1, 3, 6, 9, $15 \mathrm{~mL}$, and an unsprayed control). Two groups of bunches within each block were inoculated with $B$. cinerea, while the remaining two were left uninoculated. In order to determine $B$. cinerea incidence after the incubation period, isolations from these bunches were made onto Kerssies and paraquat mediums. From each bunch, four samples each of pedicels, rachises and receptacles were used to assess $B$. cinerea incidence. For each block and spray volume an extra bunch was included for spray cover assessment.

\section{Spray application and spray coverage assessment}

Sprays consisted of a mixture of fenhexamid (Teldor ${ }^{\circledR} 500 \mathrm{SC}$, Bayer) at the recommended dose $(75 \mathrm{~mL} / 100 \mathrm{~L})(\mathrm{Nel}$ et al., 2003) and Yellow Fluorescent Pigment ${ }^{\circledR}$ (400 g/L, EC) (South Australian Research and Development Institute, Loxton SA 5333, Australia) at $2 \mathrm{~L} / 100 \mathrm{~L}$ (Furness, 2000). In vitro tests showed that the fluorescent pigment did not influence $B$. cinerea growth on PDA medium (unpublished results). Bunches were sprayed from two sides, with spray volumes ranging from 1 to $15 \mathrm{~mL}$, by means of a gravity feed mist spray gun (ITW DEVILBISS Spray Equipment Products, 195 Internationale Blvd, Glendale Heights IL 60139 , USA) in a spray chamber $[660 \times 1410 \times 800 \mathrm{~mm}$ (h/l/w)]. These spray volumes equate to 111.11 to $1666.67 \mathrm{~L} / \mathrm{ha}$ in vineyard conditions (Furness et al., 1998). After each spray, the chamber was ventilated for 5 minutes before the next application. Following spraying, the peduncles of bunches were inserted into containers with water and kept for $24 \mathrm{~h}$ at $22^{\circ} \mathrm{C}$ before inoculation.

Spray cover assessment was done by means of the protocol developed and validated by Brink et al. (2004). Three pedicel samples for each spray treatment were taken from the sprayed side of a selected bunch. Images (12 megapixels) were digitally captured through the stereoscopic microscope at $20 \times$ magnification using a high-quality photomicrographic digital camera (Nikon DXM 1200). Image analysis was done with Image-Pro Discovery version 4.5 for Windows (Media Cybernetics, www.mediacy.com) software. Using the measurement tools, pigments within a selected area of interest (AOI), with a size of $0.465 \mathrm{~mm}^{2}$, were automatically counted and measured. The total area of deposited pigment was automatically calculated in five AOIs for each photo. Fluorescent pigment coverage was calculated as a mean of the percentage area covered by fluorescent pigment in each AOI.

\section{Inoculation and incubation}

Dry conidia from a virulent isolate of $B$. cinerea were prepared as described by Coertze and Holz (1999). Bunches were inoculated with $3 \mathrm{mg}$ dry conidia, which were dispersed by air pressure into the top of an inoculation tower (Plexiglass, $3 \times 1 \times 1 \mathrm{~m}$ [height $\times$ depth $\times$ width]) according to the method of Salinas et al. (1989). The conidia were allowed 20 minutes to settle onto the bunches that were positioned on two mesh screens on the floor of the inoculation tower. By using this inoculation technique, approximately three conidia were evenly deposited as single cells on each $\mathrm{mm}^{2}$ of bunch surface (Coertze \& Holz, 1999). Petri dishes with water agar (WA) were placed next to the bunches in the inoculation tower and the percentage germination was determined $6 \mathrm{~h}$ after inoculation (100 conidia per Petri dish, two replicates).

Following inoculation, the groups of bunches were placed on sterile epoxy-coated steel mesh screens $(53 \times 28 \times 2 \mathrm{~cm})$ in ethanol-disinfected perspex chambers (Cape Plastics, Cape Town, South Africa) lined with a sheet of chromatography paper, with the base placed in water to establish high relative humidity $(\geq 93 \%$ $\mathrm{RH})$. The bunches were incubated for $24 \mathrm{~h}$ at $22^{\circ} \mathrm{C}$. These conditions are similar to what the pathogen might encounter in nature, namely dry conidia on dry bunch parts under high relative humidity. Uninoculated bunches were used to determine the natural infection levels of $B$. cinerea.

\section{Assessment of $B$. cinerea}

From each bunch, five receptacles, pedicels and rachis sections (5 mm each) were placed on Petri dishes containing Kerssies $B$. cinerea selective medium (Kerssies, 1990), or on water agar medium supplemented with paraquat (Grindrat \& Pezet, 1994), for isolations. The plates were incubated at $22^{\circ} \mathrm{C}$ under diurnal 
light and the sections were monitored daily for symptom expression and the development of $B$. cinerea. After 11 days the number of sections yielding sporulating $B$. cinerea colonies were recorded, and these were used to determine the percentage incidence of $B$. cinerea at the various positions in the bunches. The different isolation treatments provided conditions that facilitated the development of $B$. cinerea by conidia on the surface of bunch tissue, or by mycelia in the tissue, during the period of incubation. Previous studies (Coertze \& Holz, 1999; Coertze et al, 2001; Gütschow, 2001) with grape bunch tissue on Kerssies medium showed that segments retained their active defense abilities and no superficial mycelial growth developed on the segments during the first five days of incubation. Hyphal growth usually occurred from cells underlying the cuticle into the medium after five days, which indicated direct penetration by conidia on the surface, and the development of mycelia from the host tissue during the incubation period. B. cinerea development and colony formation on segments therefore gave an indication of infection at that site as influenced by the amount of surface conidia and mycelia confronted by active defense. Paraquat terminated host resistance in the cells of the cuticular membrane without damaging host tissue (Grindrat \& Pezet, 1994), and allowed the development of conidia and mycelia on the surface, and mycelia in the tissue in the absence of active defense (Coertze \& Holz, 1999; Coertze et al, 2001).

\section{Statistical procedure}

Means of percentages fluorescent pigment cover and Botrytis incidence on pedicels, receptacles and rachises were calculated for volume, medium and stage combinations. Linear regressions were fitted on percentage cover using SAS v. 8.2 statistical software (SAS Institute, 1999, SAS Campus Drive, Cary, North Carolina 27513). Slopes were compared by using the standard error of means.

\section{RESULTS}

Germination of the inoculated $B$. cinerea conidia on water agar plates was between $86 \%$ and $96 \%$. Incidence of natural $B$. cinerea infection from uninoculated bunch parts was very low $(<1 \%)$ and the data were therefore not considered for statistical analyses.

\section{Pea size stage}

Mean fluorescent pigment coverage obtained by spray volumes 0 , $1,3,6,9$ and $15 \mathrm{~mL}$ was $0 \%, 0.13 \%, 0.51 \%, 0.75 \%, 1.15 \%$ and $1.89 \%$ respectively (Fig. 1). This was associated with a linear reduction in $B$. cinerea incidence from $46.54 \%-67.50 \%$ to 29.37 $-47.85 \%$ for the various bunch parts (Fig. 1). Good linear fits $\left(R^{2}\right.$ $=44.35 \%-90.60 \%$ ) were obtained (Table 1). Regression lines for part $\times$ medium combinations at pea size showed no significant difference between slopes, and a consistent difference in susceptibility among bunch parts was not observed (Table 1). Furthermore, significantly higher $B$. cinerea levels were recorded

\section{TABLE 1}

Equations of linear regressions lines of $B$. cinerea infection levels (y) on pedicel, receptacle and rachis at pea size stage, as determined by isolations onto Kerssies and paraquat mediums, that were fitted on percentage fluorescent pigment coverage $\left(\mathrm{x}^{\times}\right)$for different part $\times$ growth medium combinations.

\begin{tabular}{|c|c|c|c|}
\hline Part & Medium & Linear equation ( \pm Standard error)* & $\mathbf{R}^{2}$ \\
\hline Receptacle & Paraquat & $\mathrm{y}=52.69( \pm 3.351)^{\mathrm{b}}-6.80( \pm 3.426) \mathrm{x}^{\mathrm{a}}$ & $49.60 \%$ \\
\hline Pedicel & Kerssies & $\mathrm{y}=40.79( \pm 2.556)^{\mathrm{d}}-6.04( \pm 2.613) \mathrm{x}^{\mathrm{a}}$ & $57.17 \%$ \\
\hline Receptacle & Kerssies & $\mathrm{y}=40.74( \pm 3.392)^{\mathrm{d}}-6.19( \pm 3.469) \mathrm{x}^{\mathrm{a}}$ & $44.35 \%$ \\
\hline Rachis & Kerssies & $\mathrm{y}=46.54( \pm 2.078)^{\mathrm{c}}-8.40( \pm 2.114) \mathrm{x}^{\mathrm{a}}$ & $79.79 \%$ \\
\hline
\end{tabular}

*Comparison of slopes using standard error of means

\section{TABLE 2}

Equations of linear regressions lines of B. cinerea infection levels (y) on pedicel, receptacle and rachis at bunch closure stage, as determined by isolations onto Kerssies and paraquat mediums, that were fitted on percentage fluorescent pigment coverage (x) for different part $\times$ growth medium combinations.

\begin{tabular}{|c|c|c|c|}
\hline Part & Medium & Linear equation $( \pm$ Standard error)* & $\mathbf{R}^{2}$ \\
\hline Pedicel & Paraquat & $\mathrm{y}=34.60( \pm 2.495)^{\mathrm{a}}-3.54( \pm 2.938) \mathrm{x}$ ab & $26.64 \%$ \\
\hline Receptacle & Paraquat & $\mathrm{y}=28.38( \pm 2.304)^{\mathrm{b}}-4.24( \pm 2.713) \mathrm{x}^{\mathrm{ab}}$ & $36.86 \%$ \\
\hline Rachis & Paraquat & $y=28.51( \pm 1.061)^{b}+1.06( \pm 2.702) x$ & $3.71 \%$ \\
\hline Pedicel & Kerssies & $y=20.48( \pm 3.015)^{c}-3.35( \pm 3.551) x^{a b}$ & $18.21 \%$ \\
\hline Receptacle & Kerssies & $\mathrm{y}=25.00( \pm 1.283)^{\mathrm{b}}-6.22( \pm 1.511) \mathrm{x}^{\mathrm{a}}$ & $80.92 \%$ \\
\hline Rachis & Kerssies & $y=16.59( \pm 2.476)^{c}-2.31( \pm 2.916) x x^{a b}$ & $13.53 \%$ \\
\hline
\end{tabular}

*Comparison of slopes using standard error of means 


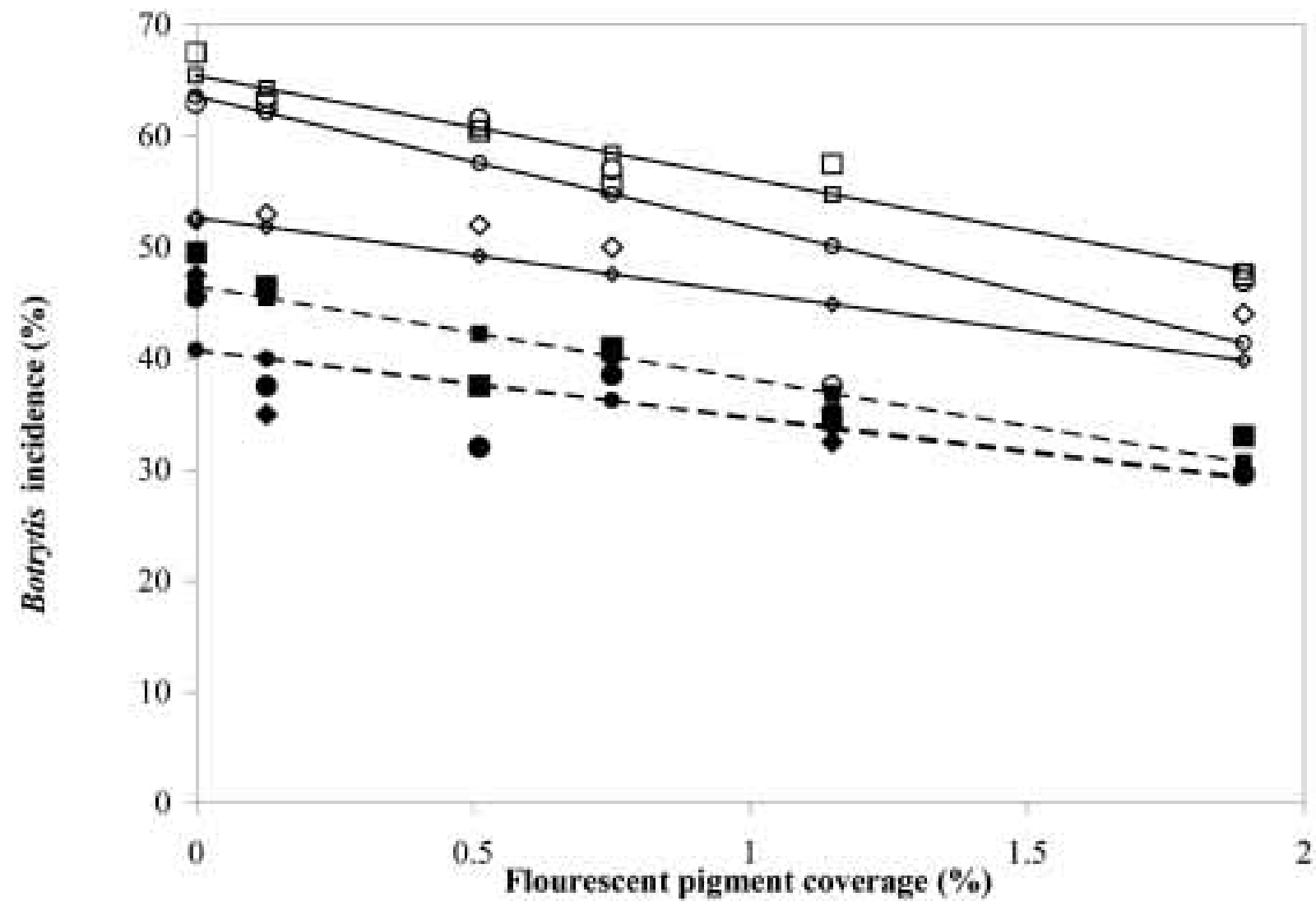

FIGURE 1

Mean percentage B. cinerea incidence on rachises (Kerssies $\square$, paraquat $\square$ ), pedicels (Kerssies $\bullet$, paraquat $\bigcirc$ ) and receptacles (Kerssies $\bullet$, paraquat $\diamond$ ) of inoculated bunches with different coverage levels resulting from fenhexamid / fluorescent pigment mixture sprayed with volumes ranging from 0 to $15 \mathrm{~mL}$ at pea size. Linear regression lines were fitted to the data: Kerssies medium (-------) and paraquat medium ( - ).

on paraquat medium $(52.69 \%-65.46 \%)$ compared to on the Kerssies medium $(40.74 \%-46.54 \%)$ on all parts of unsprayed bunches $(\mathrm{x}=0)$ (Table 1).

\section{Bunch closure}

Botrytis cinerea incidence recorded at bunch closure stage $(16.59 \%-34.60 \%)$ was markedly lower than that recorded at pea size stage $[40.74 \%-63.58 \%$ (Tables 1 and 2; Figs. 1 and 2)]. Markedly higher $B$. cinerea incidence on unsprayed bunches was recorded on paraquat medium $(28.31 \%-34.60 \%)$ compared with the Kerssies medium $(16.59 \%-25.00 \%)$ on all parts (Table 2). Mean fluorescent pigment coverage effected by spray volumes 0 , $1,3,6,9$ and $15 \mathrm{~mL}$ was markedly lower at bunch closure [0\%, $0.13 \%, 0.19 \%, 0.62 \%, 1.13 \%$ and $1.61 \%$, respectively (Fig. 2)] than at pea size (Fig. 1). A general reduction in B. cinerea from $13.5 \%-39 \%$ to $8.5-32 \%$ was observed for the various bunch parts with increased spray cover (Fig. 2). However, most combinations gave poor linear fits at bunch closure $\left(\mathrm{R}^{2}=3.71 \%-\right.$ $36.86 \%$ ), with omissible slopes for the rachis $\times$ paraquat, pedicel $\times$ kerssies and rachis $\times$ kerssies combinations (Table 2). At this stage, only the receptacle gave a favourable fit $\left(\mathrm{R}^{2}=80.92 \%\right)$. Despite the poor linear fits, similar trends and slopes were observed as those observed for pea size stage.

\section{DISCUSSION}

This study showed that an increase in fungicide spray cover reduced the infection levels of $B$. cinerea on susceptible bunch parts. This indicates the importance of adequate spray cover on susceptible bunch parts for the control of $B$. cinerea in grapevines. The linear relation between spray cover and $B$. cinerea incidence was, however, less evident at bunch closure. This might be explained by the influence of the growth stage and different bunch parts on the deposition of fungicides. The highest deposition was measured early in the season and it decreased as bunches became more compact (Brink et al., 2004). This was largely attributed to the higher porosity of bunches at pea size compared to the more compact bunches at bunch closure, as well as higher air flow retention on the front of bunches than on inner bunch parts (Barry \& Weber, 2002). It can furthermore be hypothesised that bunches at pea-size stage would have received higher dosages of evenly dispersed dry conidia than bunches at bunch closure.

Markedly higher infection levels were recorded at pea size compared with bunch closure. This can be ascribed to differences in host resistance (Holz et al., 2004). No consistent difference in susceptibility among bunch parts was observed. This is contrary to the findings of Gütschow (2001) who found pedicels to be significantly more susceptible than rachises (cultivar Dauphine). In 


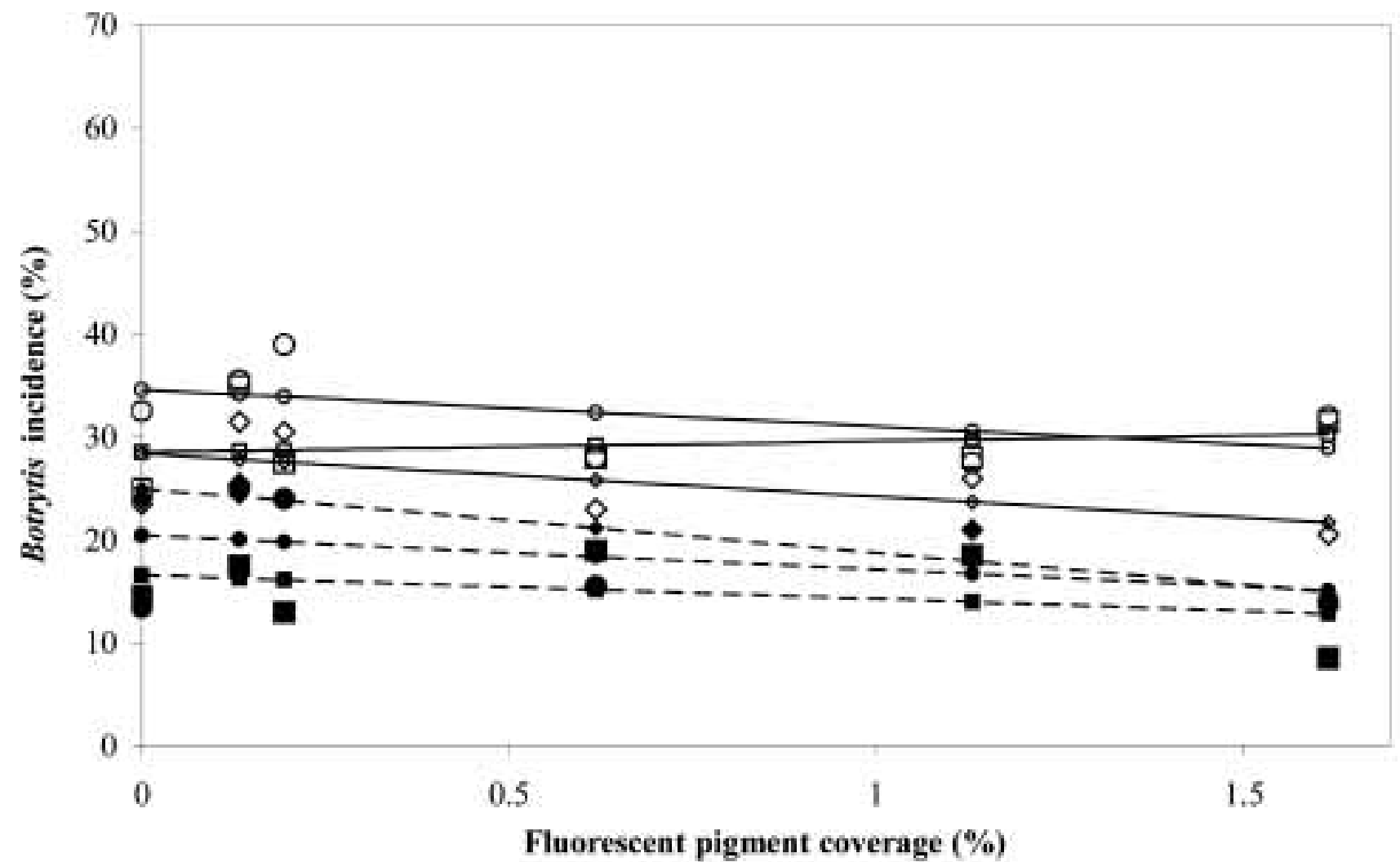

FIGURE 2

Mean percentage $B$. cinerea incidence on rachises (Kerssies $\mathbf{\square}$, paraquat $\square$ ), pedicels (Kerssies $\bullet$, paraquat $\bigcirc$ ) and receptacles (Kerssies $\bullet$, paraquat $\diamond$ ) of inoculated bunches with different coverage levels resulting from fenhexamid / fluorescent pigment mixture sprayed with volumes ranging from 0 to 15 mL at bunch closure. Linear regression lines were fitted to the data: Kerssies medium (-------) and paraquat medium ( - ).

our study, rachises might have had lower levels of fungicide cover than pedicels (Brink et al., 2004), and therefore a slightly higher incidence of $B$. cinerea.

Markedly better linear fits and higher infection levels were recorded when isolations were made on paraquat medium. Since $B$. cinerea incidence on paraquat medium was the result of infection after the termination of host resistance by the herbicide (Grindat \& Pezet, 1994), infection levels would therefore provide a clear indication of the fungicide action in the absence of active host resistance. When studying $B$. cinerea infection on Kerssies medium, fungicidal action was masked by host resistance (Coertze \& Holz, 1999; Coertze et al., 2001), which might have contributed to the variability of incidence data.

In this study, the lowest infection level (39.83\%) was achieved with the highest spray volume at pea size on the paraquat medium, compared to $0 \%$ measured by Van Rooi (2001) with the same inoculation and isolation technique, but with higher spray volumes. The spray cover levels found in this study were therefore not high enough to result in adequate control of $B$. cinerea infections.

From these findings, we conclude that an increase in spray cover will result in decreased infection. However, several factors, [i.e. too low spray volumes (i.e. spray cover), three dimensional bunches, and bunch compactness] led to inconsistent conidium and fungicide spray deposition on the structural bunch parts. Future studies aimed at determining minimum effective coverage values should use higher coverage levels to ensure adequate control of Botrytis infection. Isolations onto paraquat medium would furthermore lead to a higher and less variable assessment of $B$. cinerea infection levels. Moreover, variation in conidium and spray deposition can also be minimised by cutting bunches into two-dimensional shapes, which might lead to better linear relations between spray cover and B. cinerea infection levels.

\section{LITERATURE CITED}

Barry, S.I., Weber, R.O., 2002. The application of pesticides to grape bunches. In: Hewitt, J. (ed.). Proc. MISG 2001, Australia. pp. 28-40.

Brink, J.C., Holz, G.H., Calitz, F.J., \& Fourie, P.H., 2004. Development of a protocol to quantify spray deposits on grape bunches. 7th ISAA. ISAA 2004 Foundation, South Africa. pp. 230-236.

Coertze, S., \& Holz, G., 1999. Surface colonization, penetration, and lesion formation on grapes inoculated fresh or after cold storage with single airborne conidia of Botrytis cinerea. Plant Dis. 83, 917-924.

Coertze, S., \& Holz, G., 2002. Epidemiology of Botrytis cinerea on grape: wound infection by dry, airborne conidia. S. Afr. J. Enol.Vitic. 23, 72-77.

Coertze, S., Holz, G., \& Sadie, A., 2001. Germination and establishment of infection on grape berries by single airborne conidia of Botrytis cinerea. Plant Dis. 85, 668-677.

Derkson, R.C., \& Jiang, C., 1995. Automated detection of fluorescent spray deposits with a computer vision system. Transactions of the ASAE. 38, 16471653.

Furness, G.O., 2000. SARDI Fluorescent Pigment suspension concentrate. Fact Sheet 1-2000. SARDI. SARDI/ Primary Industries and Resources. 
Furness, G.O., Magarey, P.A., Miller, P.H., \& Drew, H.J., 1998. Fruit tree and vine sprayer calibration based on canopy size and length of row: unit canopy. Crop Prot. 17, 639-644.

Grindat, D., \& Pezet, R., 1994. Le Paraquat, un Outil pour la Révélation Rapide d' Infections Fongiques latentes et de Champignons Endophytes. J. Phytopathol. $141,86-89$

Gütschow, M., 2001. Resistance to Botrytis cinerea in parts of leaves and bunches of grapevine. Thesis, Stellenbosch University, Private Bag X1, 7602 Matieland (Stellenbosch), South Africa.

Hewitt, W.B., 1974. Rots and bunch rots of grapes. California Agricultural Experiment Station Bulletin 868.

Holz, G., Gütschow, M., Coertze, S., \& Calitz, F. J., 2003. Occurrence of Botrytis cinerea and subsequent disease expression at different positions on leaves and bunches of grape. Plant Dis. 87, 351-358.

Holz, G., \& Volkmann, A.S., 2002. Colonisation of different positions in grape bunches by potential biocontrol organisms, and the subsequent occurrence of Botrytis cinerea. In: Elad, J. Köhl, \& D. Shtienberg (eds.). IOBC/WPRS, 2002, Kusadasi, Turkey. pp. 9-12.

Holownicki, R., Doruchowski, G., Swiechowski, W. \& Jaeken, P. 2002. Methods of evaluation of spray deposit and coverage on artificial targets. Electron. J. Pol. Agric. Univ. Issue 1, 1-9.

Kerssies, A., 1990. A selective medium for Botrytis cinerea to be used in a sporetrap. Netherlands J. Plant Pathol. 96, 247-250.
Leroux, P., 1995. Progress and problems in the control of Botrytis cinerea in grapevine. Pestic. Outlook 6, 13-19.

Nair, N.G., Emmet, R.W. \& Parker, F.E., 1988. Some factors predisposing grape berries to infection by Botrytis cinerea. NZ J. Exp. Agric. 16, 257-263.

Nel, A., Krause, M. \& Khelawanalall, N. 2003. A guide for the contol of plant diseases. Government Printer: South Africa.

Salinas, J., Glandorf, D.C.M., Pacavet, F.D. \& Verhoeff, K. 1989. Effects of temperature, relative humidity and age of conidia in incidence of spotting on gerbera flowers caused by Botrytis cinerea. Neth. J. Plant Pathol. 95, 51-64.

Swart, A.E., \& Holz, G., 1994. Colonisation of table grapes bunches by Alternaria alternate and rot of cold-stored grapes. S. Afr. J. Enol. Vitic. 16, 3-6.

Thomas, C.S., Marios, J.J. \& English, J.T., 1988. The effects of wind speed, temperature and relative humidity on development of aerial mycelium and conidia of Botrytis cinerea on grape. Phytopathology 78, 260-265.

Van Rooi, C., 2001. Infection by dry, airborne Botrytis cinerea conidia and fungicide efficacy on different parts of grape bunches and vinlettes. Thesis, Stellenbosch University, Private Bag X1, 7602 Matieland (Stellenbosch), South Africa.

Van Rooi, C., \& Holz, G., 2003. Fungicide efficacy against Botrytis cinerea at different positions on grape shoots. S. Afr. J. Enol. Vitic. 24, 11-15.

Van Schoor, J., 2004. The ecology of Botrytis cinerea on grape in the Western Cape Province. Thesis, Stellenbosch University, Private Bag X1, 7602 Matieland (Stellenbosch), South Africa. 